\title{
Color-Doppler Ultrasonography in the Monitoring of Endovascular Abdominal Aortic Aneurysm Repair
}

\author{
Enrique M. San Norberto, James Taylor and Carlos Vaquero \\ Division of Vascular Surgery, Valladolid University Hospital \\ Spain
}

\section{Introduction}

The introduction of endovascular stent-graft repair for aortic aneurysm has engendered considerable enthusiasm and interest from vascular specialist. With progressive improvement in imaging, clinical experience, and stent-graft design, and the use of adjuvant procedures, a substantial number of patients are now candidates for endovascular repair of an aortic aneurysm. Endoluminal aneurysm repair, however, currently is at a critical point. Unquestionably, endoluminal aneurysm repair can reduce substantially the need for intensive care and length of hospital stay, and survival is reportedly improved when compared with open repair. Although the use of stent-grafts for the treatment of thoracic and abdominal aneurysms has increased dramatically there is little midterm or long-term proof of its efficacy. Endovascular aortic aneurysm repair (EVAR trial participants, 2005) has an initial postoperative benefit versus open AAA repair as a result of decreased early morbidity and mortality (EVAR trial participants. 2005; Lederle et al., 2007). However, as shown in EVAR trial 1 (EVAR trial participants, 2005), stent-graft-related complications are observed in approximately $40 \%$ of patients within 4 years after EVAR, resulting in a $20 \%$ reintervention rate to reduce the ongoing rupture risk. Persistent blood flow into the aneurysm sac and outside the graft lumen (endoleak) represents the most frequent complication after EVAR and is considered a procedural failure, since it is associated with aneurysm enlargement and possible rupture. The reported incidence of endoleaks ranges from $10 \%$ to $45 \%$, and lifelong surveillance is required for early detection and treatment.

As with the entire field of endovascular surgery, imaging techniques and recommendations regarding their use are changing rapidly. Only long-term follow-up data determine which methods will become standard. Currently, the imaging modalities best suited to achieve the above goals are plain film radiographs of the abdomen (chest) and CT angiography with specialized 3D reconstruction protocols (Fig. 1). In centers of excellence, color or powerDoppler ultrasound is a useful adjunctive modality and ultimately may decrease the required frequency of more expensive studies such as $\mathrm{CT}$. The modalities for postoperative imaging of endoleak may be surrogate or direct (May et al., 2005). The surrogate modalities include plain abdominal x-ray and measurement of AAA diameter by B-mode ultrasound or $\mathrm{CT}$. Because the majority of endografts have a radio-opaque metallic frame, a plain abdominal x-ray is a useful investigation (Fig. 2). It may demonstrate faulty fixation more 
clearly and earlier than contrast CT, and it may lead to the detection of endoleaks. The accuracy of detecting migration can be improved by following a protocol of performing A-P, lateral, and oblique views at the level of the umbilicus. Studies have confirmed that the presence of an endoleak is usually associated with an increase in the size of the aneurysm sac. Measurement of AAA diameter by B-mode ultrasound can therefore be used as a surrogate method of detecting endoleaks. CT may also be used for a similar purpose, with the option of monitoring an increase in volume of the sac in addition to the diameter of the sac. The direct methods of imaging for endoleaks include CDU, contrast-enhanced CT, and angiography. Contrast-enhanced $\mathrm{CT}$ has been accepted as the gold standard for detecting the presence of an endoleak. Once an endoleak has been detected, however, carefully planned arteriography is more useful in characterizing the origin and nature of the endoleak. CDU has the advantage of imaging type II endoleaks in real time, as distinct from contrast CT and arteriography, both of which have to ray on accurate timing to image the contrast arriving in the sac via collateral circulation (Prinssen, 2004).

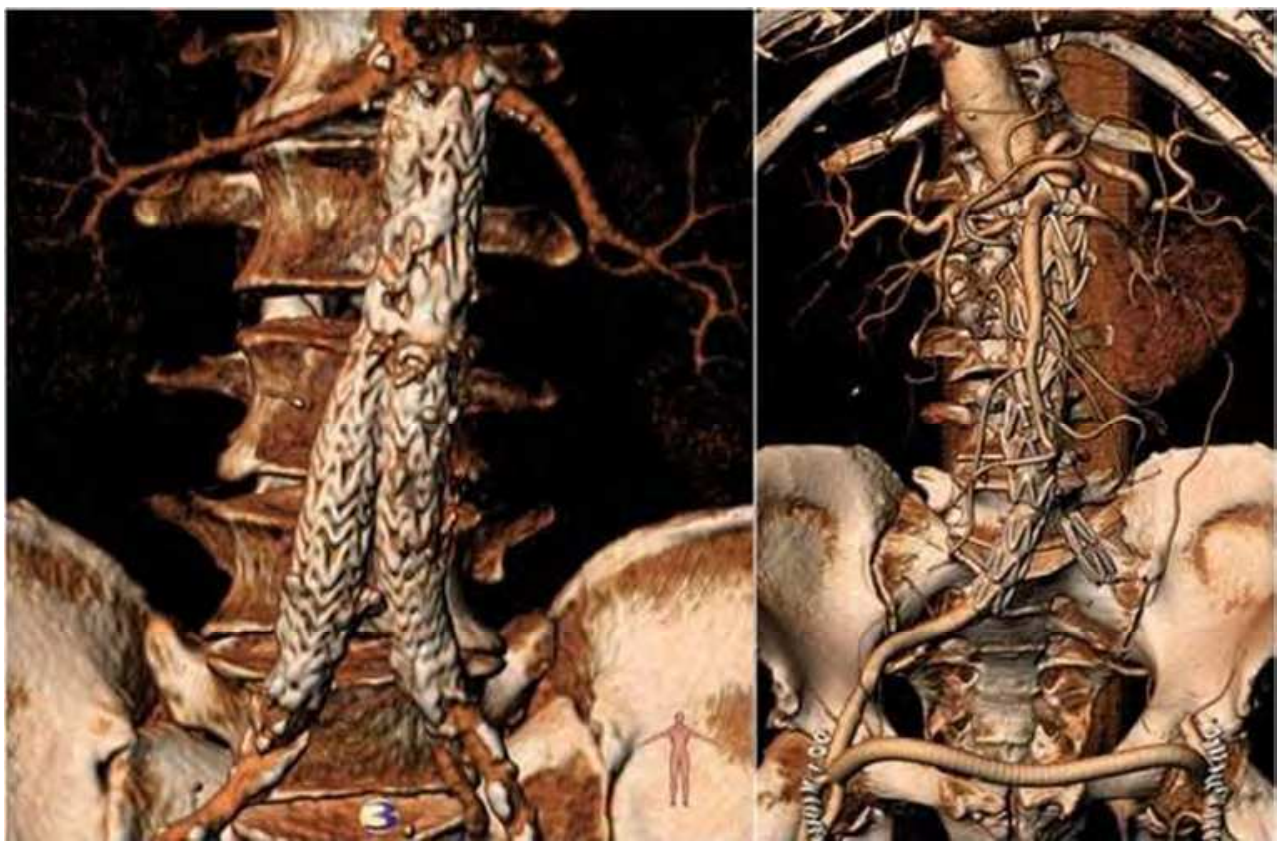

Fig. 1. CT angiography with 3D reconstruction. Left: Excluder bifurcated endoprosthesis (W.L. Gore and Associates Inc, Flagstaff, Ariz). Right: Talent aortouniiliac endoprosthesis (Medtronic Ave, St. Rosa, Cal), left common iliac occluder and femoro-femoral crossover bypass.

The goals of postprocedural imaging are to confirm and redocument the appropriate placement of the stent-graft, to assess better the effectiveness of the stent-graft in initially excluding the AAA (detecting flow in the sac), to follow the long-term fate and size of the AAA sac and ensure its stability, to detect remote stent-graft failure (structural or functional) and to better characterize and possibly treat any endoleaks. Increase in aneurysm size after EVAR is associated with an increased risk of AAA rupture and may 
require reintervention or conversion to open repair to prevent AAA rupture. Imaging evaluation should be able to show aneurismal size, changes in aneurysm size, position of stent-graft, evidence of change in position of the endoprosthesis, structural integrity of the device, endoleaks and change in the characteristics of the endoleak.

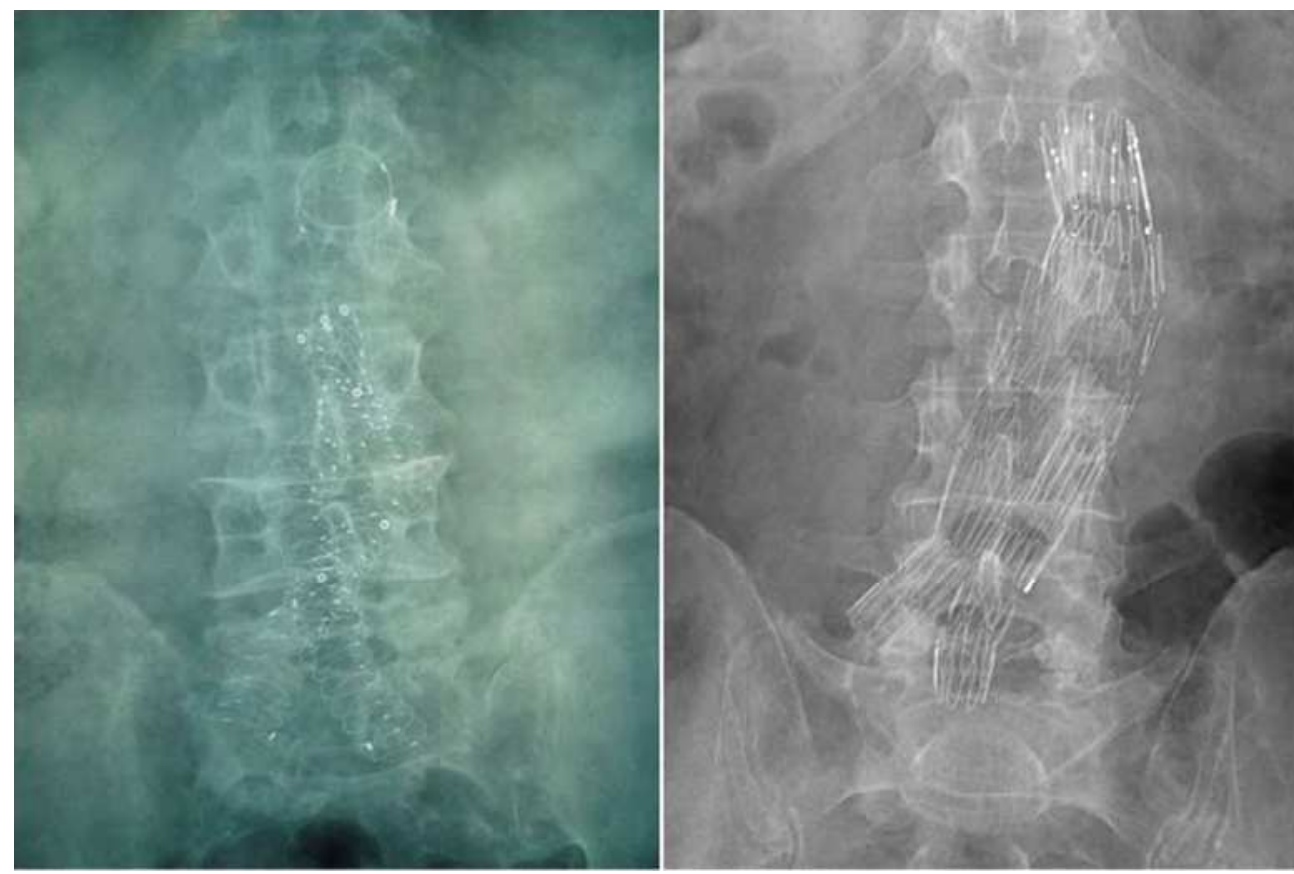

Fig. 2. Plain abdominal x-ray. Left: Anaconda bifurcated endoprosthesis (Vascutek Inc., a Terumo Company, Renfrewshire, Scotland, UK). Right: Zenith bifurcated endoprosthesis (Cook Medical Inc., Bloomington, Ind).

\section{Endoleak after EVAR}

In addition to preoperative imaging, adequate surveillance imaging modalities capable of detecting complications and treatment effects, are necessary. Follow-up imaging is directed toward repeated assessment of the aneurysm size, detection of endoleaks, and monitoring of the structural and positional integrity of the stent-graft.

An endoleak is a condition associated with endovascular stent-grafts, defined by persistent blood flow outside the lumen of the stent-graft but within the aneurysm sac or adjacent vascular segment being treated by the stent-graft. Endoleaks are usually associated with nonregresion or even expansion of the AAA. An endoleak is evidence of incomplete exclusion of the aneurysm from the circulation. There is evidence that an endoleak may resolve spontaneously, but a proportion of those that do persist are associated with late aneurysm rupture. Although intrasac pressure may approach systemical arterial pressure in the presence of an endoleak, some type II endoleaks have been associated with a decrease in aneurysm volume and intrasac pressures that are substantially less than systemic pressures (Van Sambeek, 2004). With or without an endoleak, an aneurysm that does not decrease size 
during follow-up cannot be considered to be adequately treated. Under these conditions, regular monitoring is required until definitive exclusion of the AAA is achieved. An endoleak can be classified according to the time of occurrence. An endoleak first observed during the perioperative ( $<30$ days) period is defined as a "primary endoleak", and detection thereafter is termed a "secondary endoleak". Further categorization requires precise information regarding the course of the blood flow into the aneurysm sac (Table 1). CDU could enable a better understanding of the mechanisms underlying some endoleaks and may provide more precise analysis in cases involving endoleaks due to collateral recirculation.

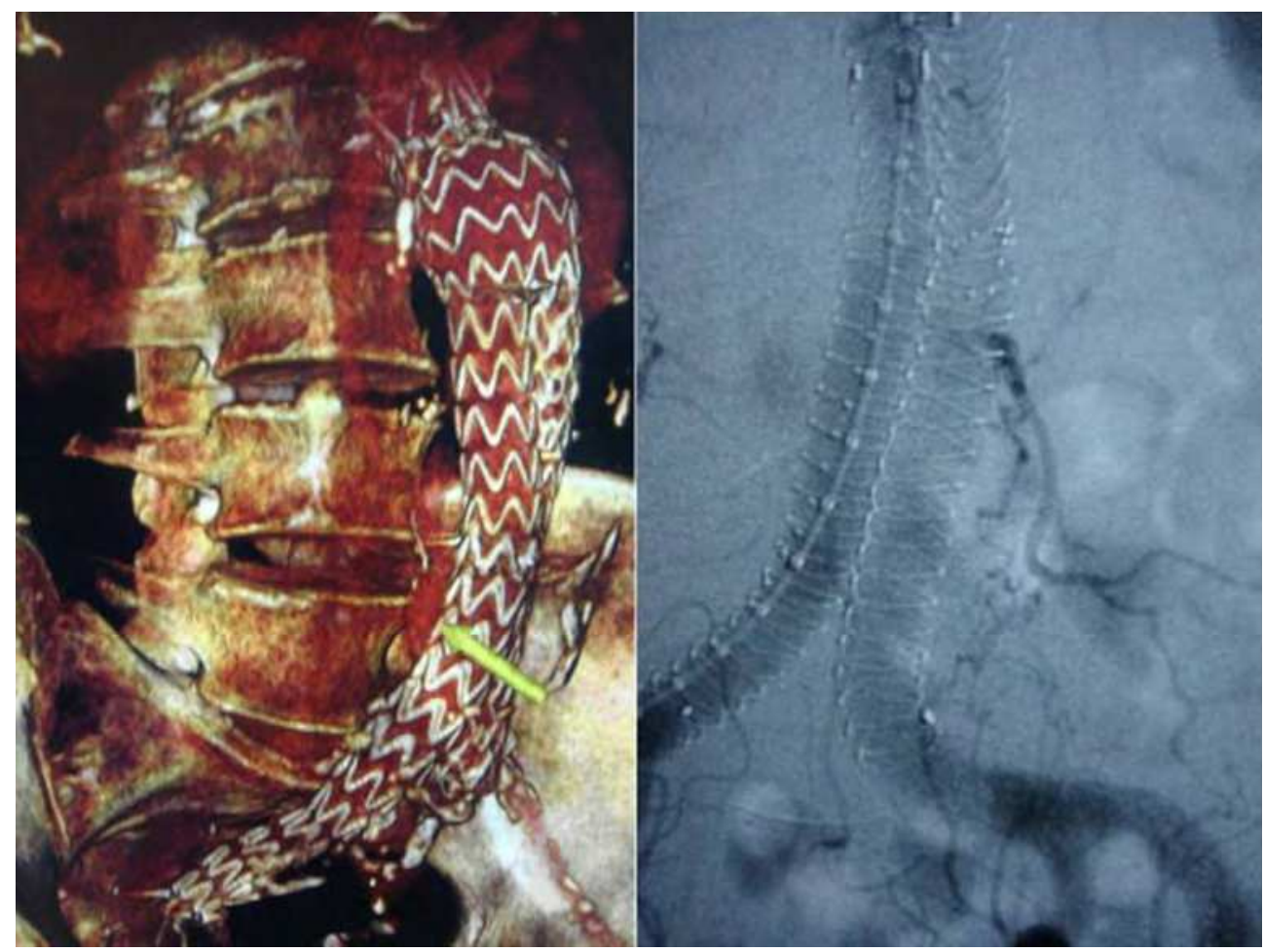

Fig. 3. Left: CT angiography with 3D reconstruction. Endurant bifurcated endoprosthesis (Medtronic Ave, St. Rosa, Cal) with a type II endoleak originated from the inferior mesenteric artery, yellow arrow. Right: Arteriography. Anaconda bifurcated endoprosthesis (Vascutek Inc., a Terumo Company, Renfrewshire, Scotland, UK) with a type II endoleak originated from a left lumbar artery.

A type I endoleak is indicative of a persistent perigraft channel of blood flow caused by inadequate seal at either the proximal (Ia) or distal (Ib) stent-graft end or attachment zones. In the case of an aorto-mono-iliac prosthesis, a type I endoleak may also refer to blood flow around an iliac occlude plug (Ic). A type II endoleak is attributed to retrograde flow from the inferior mesenteric artery (IIa), lumbar arteries (IIb), or other collateral vessels (Fig. 3). Origin and outflow sources of a type II endoleak could be specified, such as lumbar-lumbar, lumbar-inferior mesenteric artery (IMA), accessory renal-lumbar/IMA, hypogastric- 
lumbar/IMA, or undefined. It should be emphasized that any connection with a proximal or distal attachment zone will classify the endoleak as a type I endoleak. Flow hemodynamics of type II endoleaks affect the endoleak's persistence and treatment outcome. Therefore, a new, more detailed classification of leaks, particularly type II endoleak, is required that is based on Doppler waveforms and flow velocities (Fig. 4).

A type III endoleak is caused by a component disconnection (IIIa) or fabric tear, fabric disruption, or graft disintegration (IIIb). Type IIIb endoleak can be further stratified as minor $(<2 \mathrm{~mm})$ or major $(>2 \mathrm{~mm})$. A type IV endoleak is caused by blood flow through an intact but otherwise porous fabric, observed during the first 30 days after stent-graft implantation. This definition is not applicable to fabric-related endoleaks observed after the first 30-day period. Type $\mathrm{V}$ endoleaks are those in which bloodflow can be visualized within the aneurysm sac but the source cannot be identified. If an endoleak is visualized in imaging studies but the precise source cannot be determined, the endoleak is categorized as an endoleak of undefined origin. It is recognized that an AAA can continue to enlarge after endovascular repair, even in the absence of a detectable endoleak, and that this enlargement may lead to aneurysm rupture. This phenomenon is currently defined as "endotension". Explanations for persistent or recurrent pressurization of an aneurysm sac include blood flow that is below the sensitivity limits of detection with current imaging modalities or pressure transmission through thrombus or stent-graft material.

\begin{tabular}{|c|c|}
\hline Type & Cause of perigraft flow \\
\hline \multirow[t]{3}{*}{ I } & Inadequate seal at proximal end of endograft \\
\hline & Inadequate seal at distal end of endograft \\
\hline & Inadequate seal at iliac occluder plug \\
\hline II & $\begin{array}{l}\text { Flow from a collateral vessel (lumbar, IMA, accessory renal, hypogastric) } \\
\text { without attachment site connection. }\end{array}$ \\
\hline \multirow[t]{3}{*}{ III } & Flow from a modular disconnection \\
\hline & Flow from an inadequate seal at modular junction \\
\hline & Flow from a fabric disruption \\
\hline IV & Flow from porous fabric (less than 30 days after graft placement) \\
\hline $\mathrm{V}$ & Flow visualized but source unidentified \\
\hline
\end{tabular}

Table 1. Classification of endoleak. Modified from Chaikof et al., 2002.

The origins of the endoleaks were also correctly identified with duplex. Sac refilling by a lumbar artery or the IMA is readily visualized by color coding, which also determines flow direction. It is then relatively easy to differentiate a type I proximal endoleak from a type II endoleak. One difficulty, however, remains with distal attachment sites where reverse flow can also be demonstrated without aneurysm sac reperfusion and be labeled as a distal type I endoleak. CDU appears to be an excellent tool for the evaluation of high-flow endoleaks within the aneurysm sac; however, it appears limited in making the distinction between type I and type III endoleaks.

\section{Ultrasound surveillance after endovascular aneurysm repair}

The most reliable diagnostic alternative to CTA in post-EVAR life-long surveillance is still heavily debated. CDU imaging is routinely used in vascular screenings because it is easy to perform, inexpensive, portable, safe, and widely available. This technique performs poorly 
in endoleak detection, with high false-negative and false-positive results, principally due to echo reflection by the metallic portion of stent-graft, presence of calcifications, meteorism, obesity, and slow endoleak flow, which does not allow distinction of color signals coming from vessel walls and surrounding tissue from those derived from corpuscular hematic components.

The use of CDU as the preferred imaging modality in the follow-up of patients can reduce the biologic hazards associated with CT angiography (Carrafiello et al., 2008). The EVAR procedure and lifelong annual CT follow-up carry a substantial ionizing radiation burden. Patients receive a total effective dose of approximately $60 \mathrm{mSv}$ within the first year after EVAR, taking into account procedure-related fluoroscopy and follow-up CT angiography. The mean effective dose of CT angiography for EVAR follow-up is approximately $15 \mathrm{mSv}$. The stochastic risk of a fatal radiation-induced tumor is estimated to be $5 \% / \mathrm{Sv}$ radiation. Therefore, the risk of cancer induction of one CT angiography procedure is approximately 1 in 1,500 (International Commission on Radiological Protection, 2007), indicating the relevance of reliable alternatives to annual CT angiography for post-EVAR follow-up, particularly in younger patients. Second, CT angiography requires the administration of iodinated contrast agents, which are associated with nephrotoxic effects. Renal dysfunction is a comorbidity found in $80 \%$ of patients with aneurysms, and is the most important risk factor for contrast agent-induced nephrotoxicity. No major side effects, including nephrotoxic effects, have been reported for ultrasound contrast agents, which favors the use of contrast enhanced untrasound (CEUS) for post-EVAR follow-up.

The advantages of duplex ultrasound in the follow-up of patients with aortic endografts include the ability to collect accurate residual aortic sac diameter measurements serially over time. It is a very sensitive method for endoleak detection with adequate time and when a protocol is used. Ultrasound can often identify the source for endoleak classification and can readily evaluate for limb dysfunction or any other hemodynamic impairment. It is inexpensive and reproducible and requires no contrast and there may be an additive effect of CT with ultrasound in the follow-up of patients with these devices placed. The disadvantages of using duplex ultrasound may be the time commitment involved in a busy vascular laboratory. Additionally, there is a need for high-resolution equipment for the adequate performance of this examination. It is a technically challenging, subjective study that is highly dependent on the examiner and interpreter but it can be a valuable tool in the assessment of patients with aortic endografts. Ultrasound data are often influenced by the ability of the technologist as well as the quality of the equipment available. Therefore the results obtained with ultrasounds are much harder to reproduce from center to center, while $\mathrm{CT}$ can be easily standardized using a reproductible protocol for obtaining the scan. Unfortunately, interrogation of aortic endografts by either methodology can be challenging, with many subtleties to the images obtained. As a result, the best methodology for surveillance may not be one or the other, but a combination of the two. The unique ability of ultrasound to look at flow allows interrogation of the residual aneurysm sac around the endograft in ways that are likely not possible using conventional CT scans. The important aspects of endograft surveillance are the detection of endoleaks, changes in the endograft limbs, routine measurement of maximum aneurysm size and device migration.

The benefits of CT scan as an imaging modality compared with CDU imaging include that it is highly reproducible, less influenced by body habitus and offers faster image 
acquisition. However, among the limitations of CT scans is repeated radiation exposure, potential contrast-related complications, including allergy and renal insufficiency, and high cost. CDU imaging is more accurate than CT in detecting problems that threaten graft patency, such as migration, kinking, and stenosis. Color-flow images give physiologic as well as anatomic information that CT does not. CDU imaging accurately predicted all seven cases where graft patency appeared threatened. The ability to quantify and compare serial examination in a cost-effective, contrast-free, and radiation-safe manner suggests that CDU imaging should be the gold standard for EVAR limb patency follow-up. CDU imaging can almost always accurately determine if structural defects are causing a flow-related problem and graft migration. The safety of routine triphasic CT scanning for all patients undergoing follow-up post EVAR must be questioned. Although late type II endoleaks are more likely to be picked up in the delayed post-contrast phase, there is little evidence to suggest that this translates to a clinically significant advantage, in a group of patients in whom most aneurysms remain stable or shrink following treatment. Beeman et al. (2009), showed that cost savings is substantial when CDU imaging alone is used for midterm follow-up vs the accepted approach that required multiple CT scans. Bendick et al. (2003a), reported that eliminating CT as a surveillance tool after EVAR would represent a 3-year cost savings of $>\$ 16,000$ per patient. In fact, new surveillance paradigms have already been suggested to reduce the charges associated with EVAR. Kim et al. (2008), estimated that current reimbursement for long-term EVAR surveillance and secondary procedures using traditional protocols average a net loss of $\$ 2,235$ per patient.

A systematic review by Sun in 2006 was undertaken to investigate the diagnostic value of CDU compared with CT angiography for the detection of endoleaks and measurement of the aneurysm sac (the most commonly used criteria to assess the success of endovascular AAA repair). Twenty-one studies met the criteria and were included for analysis. The results are showed in table 2 . The sensitivity in the detection of endoleaks was significantly improved with contrast material-enhanced CDU compared with unenhanced CDU; however, no significant difference was found regarding the specificity, PPV, NPV, and accuracy between unenhanced and enhanced CDU. CDU was insensitive in measurement of aneurysm diameter compared with CT angiography in most situations. These results showed that CDU has not reached the diagnostic accuracy necessary to be a reliable alternative to $\mathrm{CT}$ angiography in the follow-up of endovascular AAA repair.

Iezzi et al. (2009), in the only prospective study to address this issue, shows CEUS imaging significantly improves the diagnostic performance of CDU imaging in endoleak detection in patients with endovascular aortic stent-grafts. Reported no significant difference in sensitivity for endoleak detection between analysis of arterial phase image alone, unenhanced and arterial phase images, and arterial and delayed phase images, after the initial follow-up at 1 month. Its sensitivity and negative predictive value are similar to multislice CTA $(97.5 \%$ and $97.3 \%$, respectively), and its specificity and accuracy are satisfactory $(81.8 \%$ and $89.3 \%)$ but not ideal because the false-positive rate is nearly $10 \%$. These findings support previous studies evaluating aortic stent-grafts by CEUS imaging vs CTA, where sensitivity for endoleak detection was $50 \%$ to $100 \%$, with many false-positive results. Furthermore, CEUS imaging seems to be more sensitive than CTA in diagnosing low-flow endoleaks, CTA failure may have resulted from shorter imaging duration than with CEUS imaging. 


\begin{tabular}{|cccccc|}
\hline & Sensitivity & Specificity & PPV & NPV & Accuracy \\
Unenhanced CDU & $66 \%$ & $93 \%$ & $76 \%$ & $90 \%$ & $91 \%$ \\
& $(52 \%-81 \%)$ & $(89 \%-97 \%)$ & $(65 \%-$ & $(86 \%-95 \%)$ & $(86 \%-97 \%)$ \\
& & & $87 \%)$ & & \\
Enhanced CDU & $81 \%$ & $82 \%$ & $58 \%$ & $95 \%$ & $98 \%$ \\
& $(52 \%-100 \%)$ & $(68 \%-97 \%)$ & $(26 \%-$ & $(87 \% 100 \%)$ & $(91 \%-100 \%)$ \\
& & & $90 \%)$ & & \\
CT angiography & $97.5 \%$ & $81.8 \%$ & $96.5 \%$ & $97.3 \%$ & $89.3 \%$ \\
& $(61-100 \%)$ & $(73-100 \%)$ & $(56-98 \%)$ & $(71-100 \%)$ & $(92-100 \%)$ \\
\hline
\end{tabular}

Table 2. CDU compared with CT angiography (Sun, 2006; Iezzi 2009). PPV: positive predictive value; NPV: negative predictive value.

Type 2 endoleaks are the most common endoleak following EVAR. Arko et al. reported that type 2 endoleaks intrasac flow velocities (IFV) $<80 \mathrm{~cm} /$ second were likely to resolve without treatment and that those with velocities $>100 \mathrm{~cm} /$ second were related to large branch vessel diameter and multiple endoleaks. They also suggested that higher velocity endoleaks were more resistant to transarterial embolization. Beeman et al. in 2010, found that IFV did not correlate with likelihood of closure of type 2 endoleaks, nor did high IFV predict sac enlargement. In addition, the velocity and multiplicity of type 2 endoleaks was not additive for AAA sac expansion. In other words, those with multiple branch endoleaks did not have higher velocities and, therefore, a greater chance at sac expansion post-EVAR.

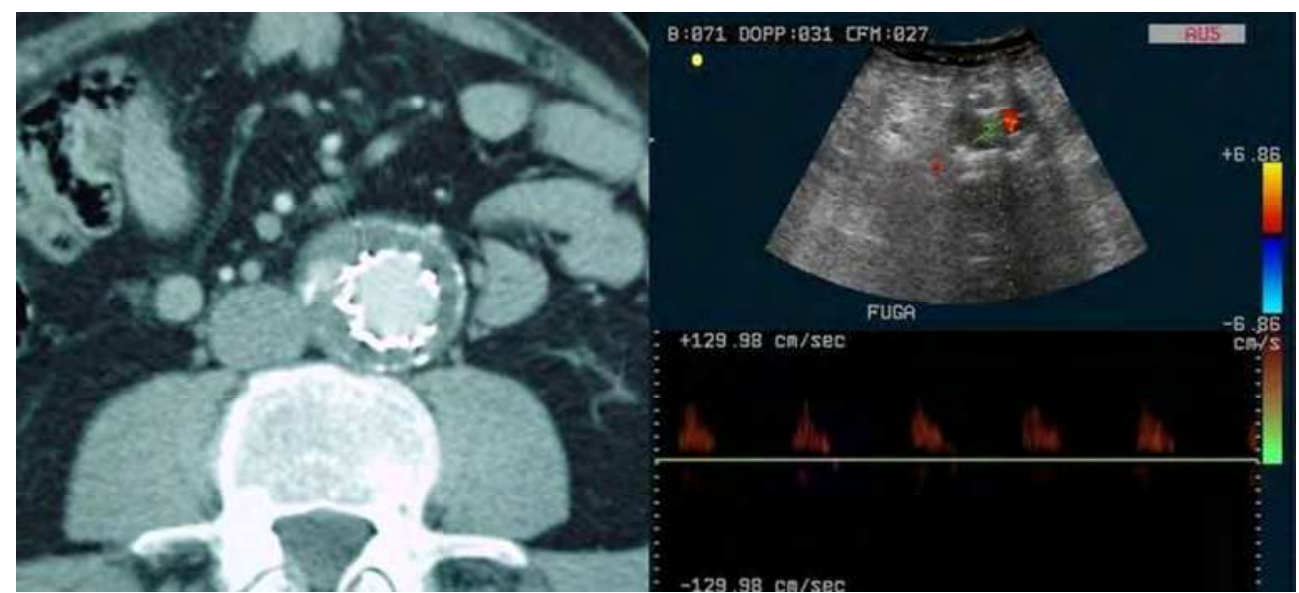

Fig. 4. An example of a type II endoleak as demonstrated on CTA. Intravenous contrast is seen outside stent-graft and within aneurysm sac. This was shown to originate from the inferior mesenteric artery, and was not associated with an increase in aneurysm sac size, and so was managed conservatively. CDU scan demonstrates the same endoleak.

Meier et al. (2001), suggested that spectral Doppler waveform (SDW) patterns can differentiate endoleaks that spontaneously seal from those that persist. They suggested that bidirectional to-from waveforms in endoleaks type 2 may precede occlusion, while 
waveforms that remain biphasic with characteristics similar to normal peripheral arterial flow appear to predict persistent endoleaks. The findings of Beeman et al. (2010) contradict their results. Bidirectional to-from waveforms could be predictive of AAA sac enlargement due to the following mechanism: if an endoleak can connect a higher pressure inflow source (lumbar or IMA) with an outflow vessel such as another nearby lower pressure lumbar vessel we would see biphasic SDWs much as normal peripheral arteries. However, if the lumbar or IMA has only an inflow source into the AAA sac and no nearby lumbar or other feeding vessel, the SDW would reveal the to-and-from SDW. The to-and-from SDW reflects the lack of an outflow source vessel and thus increases the diastolic pressure in the AAA sac; the net effect is to increase the mean pressure in the AAA sac. The presence of multiple type 2 endoleaks and bidirectional SDW may be the strongest predictive factors of increased sac diameter (Beeman et al., 2010). A to-from signal was associated with spontaneous sealing and a mono-biphasic waveform was associated with endoleak persistence (Parent et al., 2002).

\section{Imaging protocol}

The CDU is performed according to a protocol, which included the evaluation of the flow through the endograft, the perigraft flow, the renal and the iliac arterial flow, the maximum diameter of the aneurysm, and the presence of branch vessel flow. Because of the anatomy of the aneurysm stent-graft repair and the location of the endoleaks, we feel that several important aspects are essential to a complete examination of the aneurysm sac with duplex scan imaging. On the basis of the study of Sato et al., the following 4 criteria were developed to determine whether an adequate study had been performed to evaluate endoleaks:

1. A satisfactory B-mode image of the AAA sac and the stent-graft.

2. The satisfactory use of color Doppler (CD) scan imaging without an excessive overgain or undergain.

3. A CD scan assessment of the entire AAA sac outside the graft in both the transverse and the longitudinal views to screen for endoleaks.

The use of spectral Doppler scan waveform analysis outside the graft and within the AAA sac to confirm or reject potential endoleaks suggested with CD scan assessment.

All studies should be performed on a high resolution duplex ultrasound scan system with color-flow capability with technologists who are cognizant of the stent-graft structure, implantation site (aortobiiliac grafts, aortouniiliac graft with femorofemoral bypass grafting), and potential sites for endoleaks (Fig. 5). Low frequency (range, 2.25 to $5 \mathrm{MHz}$ ), curved array, phased array or mechanical sector, and pulsed Doppler scan transducers are used. Patients are studied after an overnight fast in the supine position. The graft, proximal and distal stents, and the abdominal aortic aneurysm (AAA) sac are imaged in B-mode, and size measurements of the AA sac are performed. The CD scan is added, and the settings are optimized to avoid excessive overgain (ie, color artifact that completely fills the entire color box) or undergain (ie, absence of color flow within the aortic graft). The color box size is adjusted to completely encompass the AAA sac but not made so large as to encourage artifact. The entire AAA sac outside the graft is assessed systematically by CD scan imaging in both sagittal and transverse views. Perigraft leaks are suspected with reproducible, pulsatile, $\mathrm{CD}$ scan flow images outside the graft and within the AAA sac. The focus is 
directed at the following potential leak sites: the superior and inferior stent attachments; the anterior mid-AAA sac (inferior mesenteric artery); and the posterior mid-AAA sac (lumbar arteries). A power Doppler scan may be added to assist in the detection of perigraft flow. Because spectral Doppler scan waveform will differentiate true endoleaks from color artifacts, all suspected endoleaks are evaluated by spectral Doppler scan waveform analysis. The presence of an arterial signal confirms the presence of an endoleak. Color artifacts may result from low color sensitivity settings so that pulsatility of the adjacent tissue is imaged as color signals. For all endoleaks, location, flow direction, and extent of AAA sac involvement are determined. An attempt is made to identify the origin and direction of the flow in the inferior mesenteric artery. In cases without evidence of endoleak or inadequate CD scan visualization, a systematic sampling of the AAA sac should be performed with spectral Doppler scan waveform signals to evaluate for possible endoleaks (Sato).

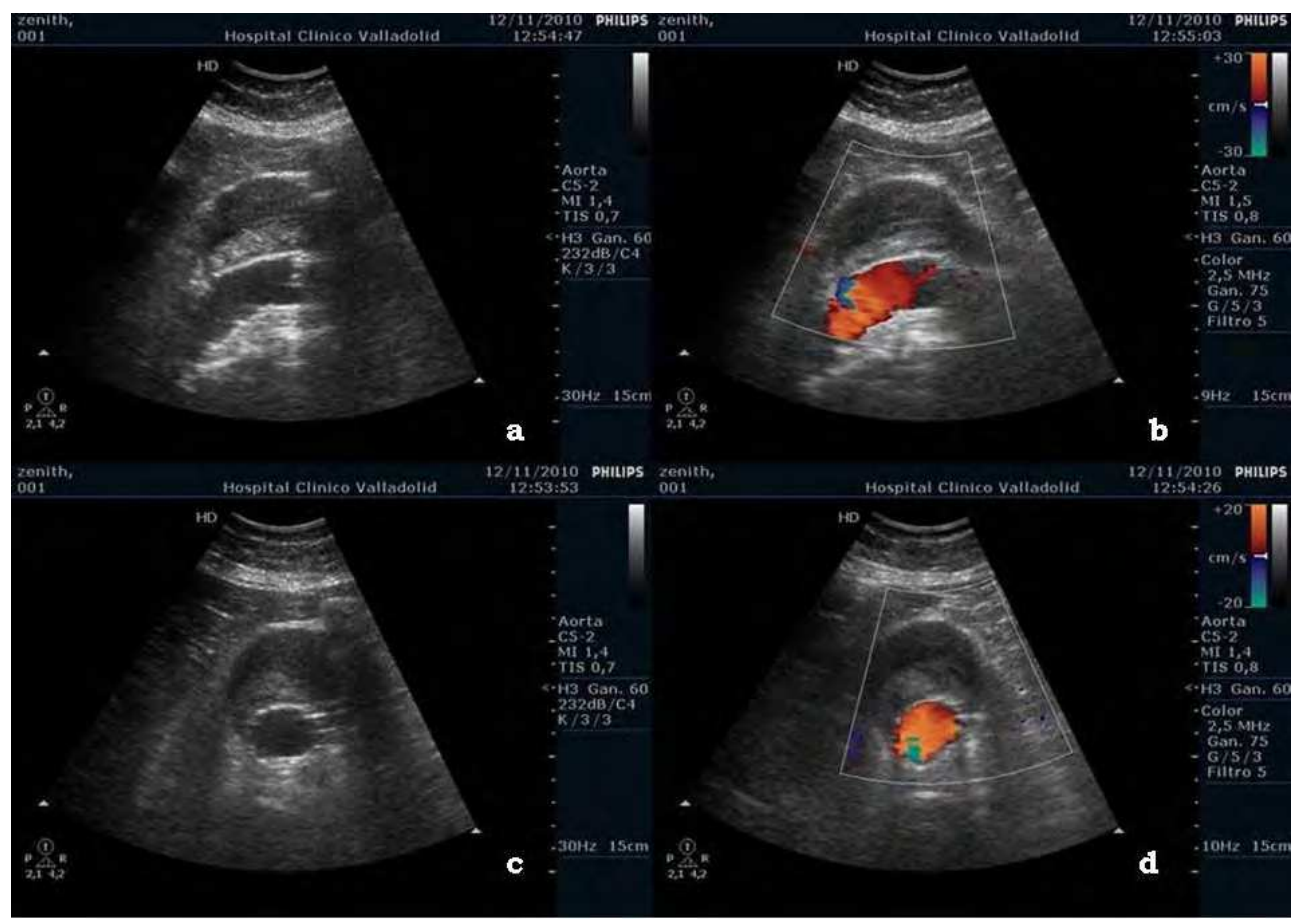

Fig. 5. Ultrasound scan imaging, main body endograft. Longitudinal view $(a, b)$ and crosssectional view $(\mathrm{c}, \mathrm{d})$ with no evidence of endoleak.

Endoleak surveillance is best performed with sensitive color-flow Doppler scale settings to show low-flow channels. These lower scale settings may produce color reverberation artifacts that obscure low-flow endoleak signals posterior to the stent-graft. Coronal views with the patient in a decubitus position may improve image quality in this posterior region. Endoleaks can be distinguished from artifacts by their reproducibility, uniform nature, and persistence during diastole when artifacts usually recede. Pulsed Doppler spectral waveform sampling of extrastent flow is used to document flow direction. Gray- 
scale images of some endoleaks are characterized by pulsatile lucencies adjacent to the stent-graft. Some, low-flow leaks may be seen as a small color spot at the stent wall during diastole. Reproducibility and persistence help differentiate these leaks from scalerelated artifacts.

A Power Doppler (PD) instrument can be helpful when the proper angle of insonation is difficult to achieve with certainty, because it is insensitive to angle considerations (Fig. 6). PD also can be helpful in low-flow situations, but care must be taken not to overinterpret artifacts caused by motion of the graft wall, which is more common in endografts that are not fully supported throughout their length. Lastly, duplex or PD can aid in the acquisition of a Doppler waveform for the flow within a suspected endoleak to analyze the direction of flow, to provide an estimate of the "resistance" of the endoleak from a nearby collateral vessel. In most cases, however, the latter information will not be of critical importance. All endoleaks related to endograft attachment sites or endograft perforations will flow from the attachment site toward a branch vessel and will have relatively low resistance and high flow. Most branch-to-branch endoleaks (ie, lumbar artery to lumbar artery), will have "to and from" waveforms. Nevertheless, any endoleak should be taken seriously no matter what the Doppler information suggests regarding its flow characteristics.

The keys to optimizing the yield of duplex ultrasonography are: have the patient prepared properly for an abdominal study; employ an experienced technologist educated about endovascular grafts, endoleaks, etc; have a physician who knows the specific endograft configuration in the room at the time of the study or available nearby; and, if possible, use contrast enhancement.

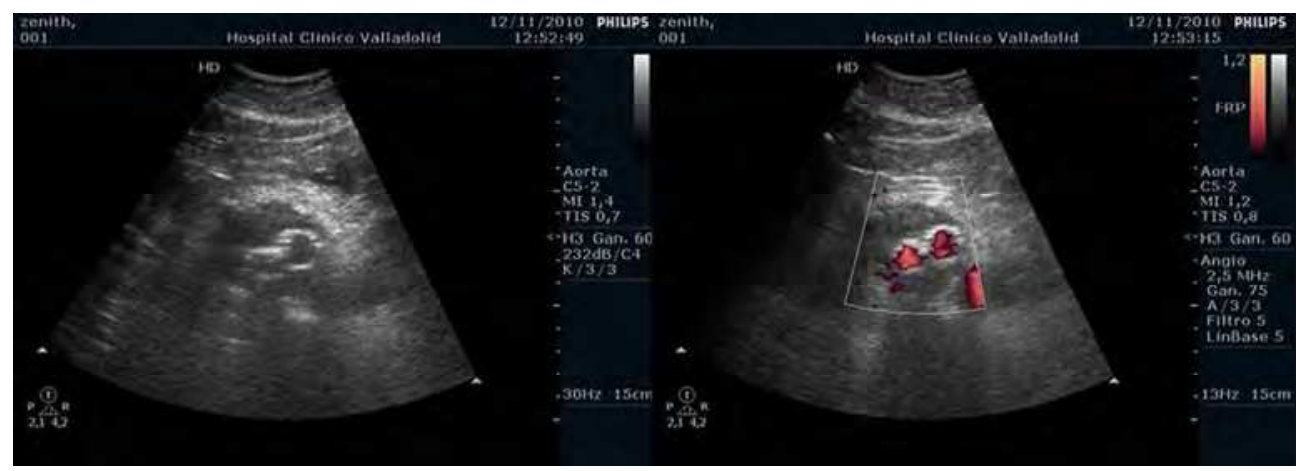

Fig. 6. Cross-sectional duplex ultrasound scan image of aortic bifurcated stent-graft with no evidence of endoleak with power-doppler imaging.

\section{Contrast-enhanced ultrasound}

A number of studies have indicated that CDU may be used for EVAR (Wolf et al., 2000; Manning et al., 2009). Non-contrast enhanced ultrasound correlates with CT angiography in determining change in AAA size over time, but has low sensitivity and positive predictive value in endoleak detection compared with conventional CT angiography (Manning et al., 2009; Elkouri et al., 2004; Raman et al., 2003). CEUS may be an alternative to CT angiography in the follow-up of patients after EVAR. As ultrasound reduces exposure to the 
biologic hazards associated with lifelong annual CT angiography, including cumulative radiation dose and nephrotoxic contrast agent load, CEUS might be considered as a substitute for CT angiography in the surveillance of patients after EVAR (Ten Bosch et al., 2010). CEUS utilizes a non-nephrotoxic contrast agent offering safer post-EVAR surveillance. However, to replace the current gold-standard for endoleak detection, any alternative imaging modality must accurately identify endoleaks with high sensitivity and specificity. In this technique, harmonic imaging is used rather than conventional grayscale. Since the ultrasound beam entrains the microbubbles to resonate at a certain frequency, imaging at that harmonic frequency results in dramatic improvements in blood flow imaging. What is sacrificed is grayscale quality; harmonic imaging loses grayscale quality as blood pool imaging is improved. Therefore the ability to evaluate blood flow is improved while the imaging of the endograft and aneurysm sac is degraded. For endoleaks, the advantage is a shortened examination with more certainty as to the presence or absence of perigraft blood flow. The combination of conventional CDU with contras-enhanced imaging when appropriate may ultimately prove to be the new standard in aortic endograft surveillance.

In this year, Mirza et al. (2010), have published a systematic review and bivariate metaanalysis about duplex ultrasound and CEUS versus computed tomography for the detection of endoleaks after EVAR. Twenty-one studies provided sufficient data for inclusion in the meta-analysis about unenhanced ultrasound. Overall, unenhanced duplex ultrasound was compared to the gold-standard of contrast-enhanced CT in 2601 patients. From the bivariate analysis, the pooled sensitivity was 0.77 and pooled specificity was 0.94 . The summary ROC curve plotted using fixed-effects meta-analysis had an area under the curve of 0.91 . Whatever, seven studies about enhanced ultrasound (285 patients), provided sufficient data for inclusion in this analysis. From the bivariate meta-analysis, the pooled sensitivity was 0.98. The pooled specificity was 0.88 . The summary ROC curve plotted using fixed-effects meta-analysis had an area under the curve of 0.96 .

There might be a relationship between blood flow characteristics and CT angiography and duplex ultrasound leakage detectability. In fact, in all patients with a nonvisualized leakage with CT angiography and CDU, the perigraft flow visualized at CEUS was characterized by very slow flow dynamics, with diffuse and delayed aneurysm enhancement. The contrast medium did not concentrate in a confined part of the sac, but it spread into the thrombus. The delayed appearance of the leak, its spreading throughout the sac, and its very slow flow could be the key factors in the explanation for the undetectability of endoleaks with CT angiography. It would be interesting to investigate whether performance of CT scanning with longer delay (>3-4 minutes after contrast agent administration) increases CT sensitivity in the detection of endoleaks in patients with enlarging aneurysms and no evidence of other complications (Napoli et al., 2004).

CEUS imaging is more specific than CTA in endoleak classification thanks to longer duration of enhancement, lack of metallic artifacts, and angio-dynamic evaluation of the leak during the dynamic phase (Fig. 7). CEUS advantages include minimal invasiveness, rapidity, good tolerability and no adverse events have been registered. On the other hand, CEUS imaging also has some limitations. Patient habitus (obesity) and bowel gas can interfere with imaging, and the patient must cooperate. The results of the ultrasound are operator-dependent, and obtaining quality images requires training and specific skills. 
Furthermore, CTA provides superior information related to graft anchoring and integrity, aneurysm morphologic changes, or visceral vessel patency (renal arteries).

\subsection{Sonographic constrast agents}

Sonographic contrast agents enhance the capability of color duplex imaging to detect endoleaks (McWilliams et al., 2002; Napoli et al., 2004). Are typically microbubbles of a perfluorocarbon gas encapsulated within a thin lipid or human albumin shell for stability while circulating in the blood pool. The microbubbles have a range of diameters from 2 to 5 microns, with a suspension of approximately $5 \times 10^{8}$ microbubbles per $\mathrm{mL}$ of fluid. The microbubbles slowly dissolve as the shell is metabolized, and the perfluorocarbon gas is eliminated through the lungs with normal respiration; a $1 \mathrm{~mL}$ bolus injection of the contrast agent will allow approximately 3 to 4 minutes of enhanced visualization and scanning time. At diagnostic ultrasound scan frequencies of 3 to $5 \mathrm{Mhz}$, the reflectivity of the microspheres is much greater than that of whole blood, increasing signal strength for imaging 100 to 1000 times.

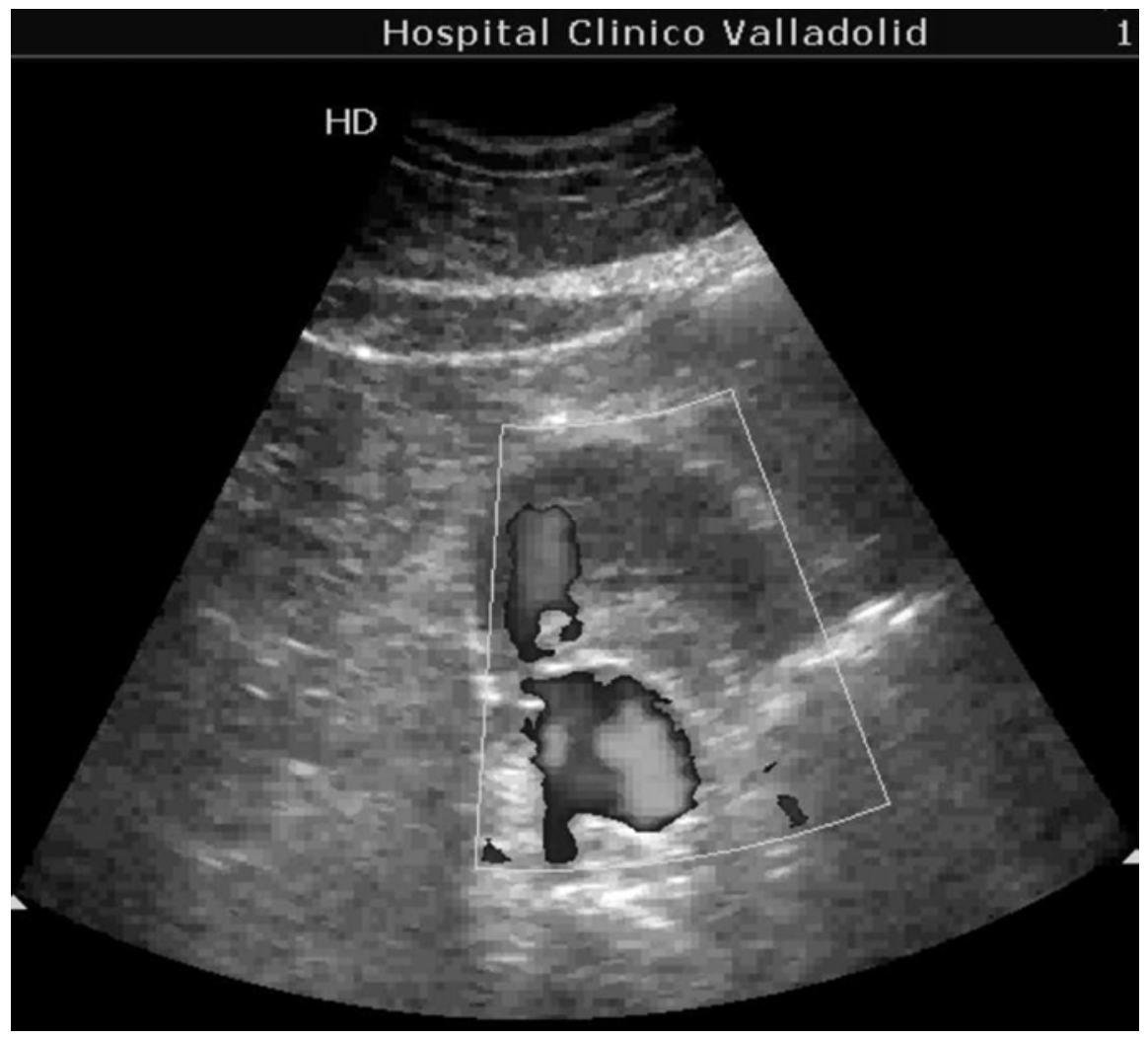

Fig. 7. CEUS demonstrating a type II endoleak. 
An additional advantage of the contrast agent is the nonlinear behavior when insonated with the ultrasound scan beam; the returning signal has strong components of both the fundamental transmitted frequency and the second harmonic, at twice the transmitted frequency. This allows imaging with the technique of tissue harmonics, which further suppresses returning echoes from stationary structures and effectively enhances the blood flow signal in the image. As applied to the surveillance of stent-grafts, it is hypothesized that increased ultrasound scan signal will arise from wherever blood is flowing, including the aneurysm sac in the case of an endoleak, with suppression of the stationary echo from the thrombus within the sac with tissue harmonic imaging to improve the contrast between these two in the resulting image (Bendick et al., 2003b). Ultrasound scan contrast agents and tissue harmonic imaging appeared less susceptible to the presence of patient obesity or bowel gas. System default settings for harmonic imaging of the contrast agent may also help eliminate some of the operator dependence in acquiring technically adequate ultrasound scans. In addition, imaging with contrast may make it easier to evaluate the position of the proximal attachment site of the stent-graft relative to an anatomic reference point, such as the origin of the real arteries, because imaging of these vessels is also enhanced with the contras agent.

Second generation sonographic contrast agents consist of stabilized microbubbles of sulphur hexafluoride gas, which is eliminated through the respiratory system, surrounded by a phospholipid shell. These microbubbles improve blood flow echogenicity by resonating with low-intensity ultrasound, which enhances backscatter and thereby increases the detected signal (Carrafiello et al., 2008). Bubble destruction during imaging is minimized, allowing real-time scanning for several minutes. No adverse events, such nephropathy, have been reported for ultrasound contrast agents. The clinical applicability of ultrasound investigation may be operator-dependent variability as well as by patient-related limitations such as obesity. There is the potential for hypersensitivity or an allergic reaction to the albumin shell of the contrast agent, which can be treated with antihistamines.

\subsection{Technique}

a. SonoVue (Bracco, Milan, Italy): A diluted sonographic contrast solution is administered by continuous intravenous infusion $(240 \mathrm{~mL} / \mathrm{h})$ for a period of 15 minutes. Sonographic contras solution is obtained by mixing $5 \mathrm{~mL}$ SonoVue containing $8 \mu \mathrm{l}$ sulfur hexafluoride microbubbles per millimeter with $55 \mathrm{~mL}$ saline solution. Continuous real time tissue harmonic imaging for endoleak detection was performed for 15 minutes during sonographic contrast agent infusion at a mechanical index for 0.4-0.5 at low acoustic power (Ten Bosch et al., 2010). According to the package insert recommendation of the manufacturer, in which the optimal dose for vascular examination was fixed at $2.4 \mathrm{~mL}$, in which a lower dose $(1.5 \mathrm{~mL})$ appeared to be sufficient for endoleak detection in the majority of patients. The agent is administered into an antecubital vein at a dose of $1.5-2.4 \mathrm{~mL}$, followed by a flush of $5 \mathrm{~mL}$ saline solution.

b. Levovist (Schering Company, Berlin, Germany): which contains $99.9 \%$ D-galactose and $0.1 \%$ palmitin acid, is a crystalline powder that is mixed with injectable water. In this suspension, the microparticles bind tiny air bubbles, enhancing the Doppler signal quality when injected intravenously by up to $25 \mathrm{~dB}$ without increasing 
background noise. This is a clear improvement in the signal-to-noise ratio (Heilberger et al., 1997).

c. Optison (Mallinckrodt, Saint Louis, Missouri): a $1 \mathrm{~mL}$ bolus of ultrasound scan contrast agent is given via injection into an antecubital vein, followed by a flush of $5 \mathrm{~mL}$ of normal saline solution. The contrast agent is allowed to circulate in the blood pool for approximately 1 minute, and then the aortic stent-graft and aneurysm sac were again scanned from the level of the diaphragm to below iliac limb attachment points in longitudinal and cross-sectional scanning modes (Bendick et al., 2003b).

\section{Color duplex ultrasound limitations}

The major disadvantages of the CDU are related to the proper instrumentation and to technologist dependency. In addition, satisfactory images cannot be obtained in some patients because of technical inability (ie, obesity or excessive intestinal gas). A review of the CDU studies from multiple centers for the EnACT stent-graft trial (Sato et al., 1998), revealed that most of the studies were suboptimal in the evaluation for endoleaks. Although approximately $90 \%$ of the studies were technically possible, as indicated with satisfactory Bmode images, complete evaluation for endoleaks as outlined in the methods described was obtained in only $19 \%$ of the studies. The most glaring deficiencies were the failure to assess the entire AAA sac with CD scan imaging and the failure to use spectral Doppler scan waveform analysis outside the graft but within the AAA sac to confirm suspected endoleaks. Errors in technical settings could be identified in some cases (ie, poor gain or focal zone settings). Most of the incomplete CDU studies for endoleaks appeared to be the result of a lack of a standardized protocol for the technologist to improve the accuracy for endoleak diagnosis with CDU technology. Others have shown improved sensitivity of CDU in identifying endoleaks when intravenous ultrasound scan contras agents are added to the study.

Ultrasound examinations may carry a number of well known limitations. First, operator dependency might limit reproducibility of the results. It is clearly true that assessment of a CT scan is less operator-dependent. Unfortunately, interobserver variability for endoleak detection by CEUS could not be assessed because this would require repetitive sonographic contrast agent infusions. Second, patient habitus may interfere with ultrasound imaging, as patient obesity or bowel gas. CDU imaging with contrast may prove to be especially useful for obese patients but is not necessarily any better in most patients, especially considering the extra cost and more difficult technique required to use this method.

A potential drawback of CEUS is the inability to detect kinking and migration of the stentgraft. Plain abdominal radiography may be used for the detection of graft migration and structural failure. In addition, sonographic contrast agents are not available worldwide yet, which may limit the applicability of CEUS techniques. Interobserver variability in technical factors can be another important limitation in the diagnostic value of CDU imaging. Furthermore, ultrasound scanning measurements have been noted to underestimate the true size of the AAA when compared with CT. Nevertheless, there is a good correlation between CDU and CT in determining aneurysm size changes over time.

Even if the technologists are very experienced, the use of relatively older equipment and short scan times may be the main culprit in an inadequate correlation between CDU and CT 
in the detection of endoleaks. Most large hospital laboratories have not uniformly upgraded to newer equipment. Therefore, it is essential that clinical decisions based on CDU be undertaken only after a review of local results indicates equivalent or superior results with this modality when compared to CT scanning. Congested waiting lists for vascular studies and an inability to attract enough trained technologists have limited the ability of hospital laboratories to devote longer time periods to each study. The lack of additional diagnostic utilities of CDU over CT scanning under these conditions has led to abandon CDU as a routine test for EVAR surveillance. Based on literature, conditions in which ultrasound imaging alone can be proposed for EVAR follow-up can be listed as follows (Long et al., 2005)::

- High quality of technical conditions of ultrasound imaging: such examination requires at least from medium- to high-end ultrasound scanner.

- High quality of medical conditions of ultrasound imaging: it must be performed by a trained senior operator really involved in AAA follow up and having an accurate knowledge of AAA endovascular treatment. Precise data concerning preoperative AAA diameter, nature of stent-graft, operative report, and events before discharge and during follow-up, such as complementary procedures, must be clearly communicated.

- Previous ultrasound imaging evaluation: evaluation of ultrasound performance for AAA diameter measurement and endoleak detection (especially type I) compared with CT remains essential in each ultrasound laboratory. A common protocol for measurements of AAA diameter with ultrasound and CT is highly recommended.

- Selection of patients: patients must be good candidates for ultrasounds. Poor echogenicity may represent a transient or definitive contraindication for ultrasound follow-up; it should be determined by the operator himself; a score of ultrasound image quality as proposed by Sato could be established.

- Absence of type I endoleak: a type I endoleak at discharge or during follow-up is a definitive contraindication for ultrasound follow-up, until the real utility is proven for ultrasound performance;

- Retraction of AAA: a clear decrease in AAA diameter diagnosed with ultrasound or unenhanced CT studies allows for ultrasound alone follow-up until suspicion of regrowth.

\section{Conclusions}

The use of endovascular techniques to repair AAAs has gained wide acceptance in the surgical community. Disadvantages of endovascular repair include late complications and the need for long-term surveillance to monitor possible sequelae, such as endoleaks, migration, aneurismal dilatation, or possible graft thrombosis. The optimal follow-up of patients after EVAR is still unknown and the repeated exposure to radiation, inconvenience for patients, and cost involved are important. The modality for long-term follow-up has been debated and there is a need for accurate, cost-effective means of postoperative surveillance.

Ultrasound offers the advantages of low cost and lack of radiation exposure. High-quality ultrasound reliably excludes endoleaks in patients after stent-grafting of AAA. Although 
duplex ultrasound is often used to augment CT scanning in post-EVAR follow-up, evidence suggest it is unsuitable for sole use in endoleak detection after EVAR. As ultrasound precludes the risks of contrast-induced nephropathy and ionizing radiation load, CEUS might be considered as a substitute to $\mathrm{CT}$ angiography as the primary imaging modality in the surveillance of patients after EVAR, provided those patients are suitable for abdominal/pelvic ultrasound and highly trained ultrasound operators are available. CEUS offers promise as a safe and sensitive modality for endoleak detection, potentially obviating the need for patient exposure to high radiation doses and nephrotoxic agents in recurrent CT imaging. Combining CDU scan with a noncontrast CT and abdominal x-ray is a useful strategy in patients who cannot have radio-contrast. Using both test permits assessment about endoleaks along with aneurysm size, seal zone, and possible graft migration. CEUS imaging is a fast, noninvasive, reliable, and valid alternative to multislice CTA for endoleak detection in endovascular aortic stent-graft patients, and is superior to unenhanced ultrasound imaging.

\section{References}

Arko, F.R.; Filis, K.A.; Siedel, S.A.; Johnson, B.L.; Drake, A.R.; Fogarty, T.J. \& Zarins, C.K. (2003). Intrasac flow velocities predict sealing of type II endoleaks after endovascular abdominal aortic aneurysm repair. Journal of Vascular Surgery, Vol. 37, No. 1 (January 2003), pp. 8-15, ISSN 0741-5214.

Beeman, B.R.; Doctor, L.M.; Doerr, K.; McAfee-Bennett, S.; Dougherty, M.J. \& Calligaro, K.D. (2009). Duplex ultrasound imaging alone is sufficient for midterm endovascular aneurysm repair surveillance: A cost analysis study and prospective comparison with computed tomography scan. Journal of Vascular Surgery, Vol. 50, No. 5, (November 2009), pp. 1019-1024, ISSN 0741-5214.

Beeman, B.R.; Murtha, K.; Doerr, K.; McAfee-Bennett, S.; Dougherty, M.J. \& Calligaro, K.D. (2010). Duplex ultrasound factor predicting persistent type II endoleak and increasing AAA sac diameter after EVAR. Journal of Vascular Surgery, Vol. 52, No. 5, (November 2010), pp. 1147-1152, ISSN 0741-5214.

Bendick, P.J.; Bove, P.G.; Long, G.W.; Zelenock, G.B.; Brown, O.W. \& Shanley, C.J. (2003). Efficacy of ultrasound scan contrast agents in the noninvasive follow-up of aortic stent-grafts. Journal of Vascular Surgery, Vol. 37, No. 2, (February 2003), pp. 381-385, ISSN 0741-5214.

Bendick, P.J.; Zelenock, G.B.; Bove, P.G.; Long, G.W.; Shanley, C.J. \& Brown, O.W. (2003). Duplex ultrasound imaging with an ultrasound contrast agent: the economic alternative to CT angiography for aortic stent-graft surveillance. Vascular and Endovascular Surgery, Vol. 37, No. 3, (May-June 2003), pp. 165-170, ISSN 15385744 .

Carrafiello, G.; Recaldini, C.; Laganà, D.; Piffaretti, G. \& Fugazzola, C. (2008) Endoleak detection and classification after endovascular treatment of abdominal aortic aneurysm: value of CEUS over CTA. Abdominal Imaging, Vol. 33, No. 3, (May-June 2008), pp. 357-362, ISSN 0942-8925.

Chaikof, E.L.; Blankensteijn, J.D.; Harris, P.L.; Zarins, C.K.; Bernhard, V.M.; Matsumura, J.S.; May, J.; Veith, F.J.; Fillinger, M.F.; Rutherford, R.B. \& Kent, K.C. Ad Hoc 
Committee for Standarized Reporting Practices in Vascular Surgery of the Society for Vascular Surgery/ American Association for Vascular Surgery. (2002) Reporting standards for endovascular aortic aneurysm repair. Journal of Vascular Surgery, Vol. 35, No. 5, (May 2002), pp. 1048-1060, ISSN 0741-5214.

Elkouri, S.; Panneton, J.M.; Andrews, J.C.; Lewis, B.D.; McKusick, M.A.; Noel, A.A.; Rowland, C.M.; Bower, T.C.; Cherry, K.J. Jr \& Glovicki, P. (2004). Computed tomography and ultrasound in follow-up patients after endovascular repair of abdominal aortic aneurysm. Annals of Vascular Surgery, Vol. 18, No. 3, (May 2004), pp. 271-279.

EVAR trial participants. (2005). Endovascular aneurysm repair versus open repair in patients with abdominal aortic aneurysm (EVAR trial 1): randomized controlled trial. Lancet, Vol. 365, No. 9478, (June-July 2005), pp. 2179-2186, ISSN 0140-6736.

Heilberger, P.; Schunn, C.; Ritter, W.; Weber, S. \& Raithel D. (1997). Postoperative color flow duplex scanning in aortic endografting. Journal of Endovascular Surgery, Vol. 4, No. 3, (August 1997), pp. 262-271, ISSN 1074-6218.

Iezzi, R.; Basilico, R.; Giancristofaro, D.; Pascali, D.; Cotroneo, A.R. \& Storto, M.L. (2009). Contrast-enhanced ultrasound versus color duplex ultrasound imaging in the follow-up of patients after endovascular abdominal aortic aneurysm repair. Journal of Vascular Surgery, Vol. 49, No. 3, (March 2009), pp. 552-560, ISSN 0741-5214.

Kim, J.K.; Tonnessen, B.H.; Noll, R.E. Jr; Money, S.R. \& Sternbergh, W.C. 3 ${ }^{\text {rd }}$ (2008). Reimbursement of long-term postplacement cost after endovascular abdominal aortic aneurysm repair. Journal of Vascular Surgery, Vol. 48, No. 6 (December 2008), pp. 1390-1395, ISSN 0741-5214.

Lederle, F.A.; Kane, R.L.; MacDonald, R. \& Wilt, T.J. (2007) Systematic review: repair of unruptured abdominal aortic aneurysm. Annals of Internal Medicine, Vol. 146, No. 10, (May 2007), pp. 735-741, ISSN 0003-4819.

Long A, Louail B, Turmel-Rodrigues L, Julia P, Sapoval M. (2005). EVAR surveillance: is ultrasound imaging alone reliable in routine practice? In: Controversies and updates in vascular surgery, Becquemin JP, Alimi YS, Wateletbe J, pp. (8-16), Edizioni Minerva Medica, 88-7711-488-6, Turin.

Manning, B.J.; O’Neill, S.M.; Haider, S.N.; Colgan, M.P.; Madhavan, P. \& Moore, D.J. (2009). Duplex ultrasound in aneurysm rurveillance following endovascular aneurysm repair: a comparison with computed tomography aortography. Journal of Vascular Surgery, Vol. 49, No. 1, (January 2009), pp. 60-65, ISSN 0741-5214.

May J, Harris JP, Kidd J, White GH. (2005). Imaging modalities for the diagnosis of endoleak, In: Vascular diagnosis, Mansour MA, Labropoulos N, pp. (407-19), Elsevier, 0-7216-9426-8, Philadelphia.

McWilliams, R.G.; Martin, J.; White, D.; Gould, D.A., Rowlands, P.C.; Haycox, A.; Brennan, J.; Gilling-Smith, G.L. \& Harris, P.L. (2002). Detection of endoleak with enhanced ultrasound imaging: comparison with biphasic computed tomography. Journal of Endovascular Therapy, Vol. 9, No. 2, (April 2002), pp. 170-179.

Meier, G.H.; Parker, F.M.; Godziachvili, V.; Demasi, R.J.; Parent, F.N. \& Gayle, R.G. (2001). Endotension after endovascular aneurysm repair: the Ancure experience. Journal of Vascular Surgery, Vol. 34, No. 3, (September 2001), pp. 426-427, ISSN 0741-5214. 
Mirza, T.A.; Karthikesalingam, A.; Jackson, D.; Walsh, S.R.; Holt, P.J.; Hayes, P.D. \& Boyle, J.R. (2010). Duplex ultrasound and contrast-enhanced ultrasound versus computed tomography for the detection of endoleak after EVAR: systematic review and bivariate meta-analysis. European Journal of Vascular and Endovascular Surgery, Vol. 39, No. 4, (April 2010), pp. 418-428, ISSN 1078-5884.

Napoli, V.; Bargellini, I.; Sardella, S.G.; Petruzzi, P.; Cioni, R.; Vignali, C.; Ferrari, M. \& Bartolozzi, C. (2004) Abdominal aortic aneurysm: contrast enhanced US for missed endoleaks after endoluminal repair. Radiology, Vol. 233, No. 1, (October 2004), pp. 217-225, ISSN 0033-8419.

Parent, F.N.; Meier, G.H.; Godziachvili, V.; LeSar, C.J.; Parker, F.M.; Carter, K.A.; Gayle, R.G.; DeMasi, R.J.; Marcinczyk, M.J. \& Gregory, R.T. (2002). The incidence and natural history of type I and II endoleak: a 5-year follow-up assessment with color duplex ultrasound scan. Journal of Vascular Surgery, Vol. 35, No. 3, (March 2002), pp. 474-481, ISSN 0741-5214.

Prinssen, M.; Verhoeven, E.L.; Buth, J.; Cuypers, P.W.; van Sambeek, M.R.; Balm, R.; Buskens, E.; Grobbee, D.E. \& Blankensteijn, J.D.; Dutch Randomized Endovascular aneurysm Management (DREAM) Trial Group. (2004) A randomized trial comparing conventional and endovascular repair of abdominal aortic aneurysms. New England Journal of Medicine, Vol. 351, No. 16, (October 2004), pp. 1607-1618, ISSN 0028-4793.

Raman, K.G.; Missig-Carroll, N.; Richardson, T.; Muluk, S.C. \& Makaroun, M.S. (2003). Color-flow duplex ultrasound scan versus computed tomographic scan in the surveillance of endovascular aneurysm repair. Journal of Vascular Surgery, Vol. 38, No. 4, (October 2003), pp. 645-651, ISSN 0741-5214.

Sato, D.T.; Goff, C.D.; Gregory, R.T.; Robinson, K.D.; Carter, K.A.; Herts, B.R.; Vilsack, H.B.; Gayle, R.G.; Parent, F.N. 3ri; DeMasi, R.J. \& Meier, G.H. (1998). Endoleak after aortic stent graft repair: diagnosis by color duplex ultrasound scan versus computed tomography scan. Journal of Vascular Surgery, Vol. 28, No. 4, (October 1998), pp. 657-663, ISSN 0741-5214.

Sun Z. (2006). Diagnostic value of color duplex ultrasonography in the follow-up of endovascular repair of abdominal aortic aneurysm. Journal of Vascular Interventional Radiology, Vol. 17, No.5, (May 2006), pp. 759-764, ISSN 1051-0443.

Ten Bosch, J.A.; Rouwet, E.V.; Peters, C.T.; Jansen, L.; Verhagen, H.J.; Prins, M.H. \& Teijnk, J.A. (2010). Contrast-enhanced ultrasound versus computed tomographic angiography for surveillance of endovascular abdominal aortic aneurysm repair. Journal of Vascular Interventional Radiology, Vol. 21, No. 5, (May 2010), pp. 638-643, ISSN 1051-0443

The 2007 Recommendations of the International commission on Radiological Protection. ICRP publication 103. Annals of the ICRP, Vol. 37, No. 2-4, (2007), pp. 1-332, ISSN 0146-6453.

Van Sambeek, MRHM. (2004). Abdominal Aneurysms - EVAR, In: Comprehensive Vascular and Endovascular Surgery, Hallett JW, Mills JL, Earnshaw JJ, Reekers JA, pp. (409423), Mosby, 0-7234-3232-5, Edinburgh. 
Wolf, Y.G.; Johnson, B.L.; Hill, B.B.; Rubin, G.D.; Fogarty, T.J. \& Zarins, C.K. (2000). Duplex ultrasound scanning versus computed tomographic angiography for postoperative evaluation of endovascular abdominal aortic aneurysm repair. Journal of Vascular Surgery, Vol. 32, No. 6, (December 2000), pp. 1142-1148, ISSN 0741-5214. 


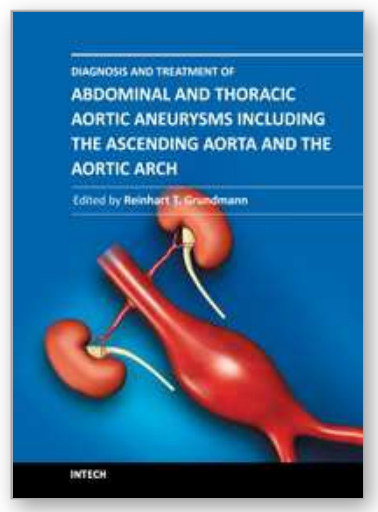

\author{
Diagnosis and Treatment of Abdominal and Thoracic Aortic \\ Aneurysms Including the Ascending Aorta and the Aortic Arch
}

Edited by Prof. Reinhart Grundmann

ISBN 978-953-307-524-2

Hard cover, 208 pages

Publisher InTech

Published online 22, June, 2011

Published in print edition June, 2011

This book considers diagnosis and treatment of abdominal and thoracic aortic aneurysms. It addresses vascular and cardiothoracic surgeons and interventional radiologists, but also anyone engaged in vascular medicine. The book focuses amongst other things on operations in the ascending aorta and the aortic arch. Surgical procedures in this area have received increasing attention in the last few years and have been subjected to several modifications. Especially the development of interventional radiological endovascular techniques that reduce the invasive nature of surgery as well as complication rates led to rapid advancements. Thoracoabdominal aortic aneurysm (TAAA) repair still remains a challenging operation since it necessitates extended exposure of the aorta and reimplantation of the vital aortic branches. Among possible postoperative complications, spinal cord injury (SCl) seems one of the most formidable morbidities. Strategies for TAAA repair and the best and most reasonable approach to prevent SCI after TAAA repair are presented.

\title{
How to reference
}

In order to correctly reference this scholarly work, feel free to copy and paste the following:

Enrique M. San Norberto, James Taylor and Carlos Vaquero (2011). Color-Doppler Ultrasonography in the Monitoring of Endovascular Abdominal Aortic Aneurysm Repair, Diagnosis and Treatment of Abdominal and Thoracic Aortic Aneurysms Including the Ascending Aorta and the Aortic Arch, Prof. Reinhart Grundmann (Ed.), ISBN: 978-953-307-524-2, InTech, Available from: http://www.intechopen.com/books/diagnosis-andtreatment-of-abdominal-and-thoracic-aortic-aneurysms-including-the-ascending-aorta-and-the-aorticarch/color-doppler-ultrasonography-in-the-monitoring-of-endovascular-abdominal-aortic-aneurysm-repair

\section{INTECH}

open science | open minds

\section{InTech Europe}

University Campus STeP Ri

Slavka Krautzeka 83/A

51000 Rijeka, Croatia

Phone: +385 (51) 770447

Fax: +385 (51) 686166

www.intechopen.com

\section{InTech China}

Unit 405, Office Block, Hotel Equatorial Shanghai

No.65, Yan An Road (West), Shanghai, 200040, China 中国上海市延安西路65号上海国际贵都大饭店办公楼 405 单元

Phone: +86-21-62489820

Fax: +86-21-62489821 
(C) 2011 The Author(s). Licensee IntechOpen. This is an open access article distributed under the terms of the Creative Commons Attribution 3.0 License, which permits unrestricted use, distribution, and reproduction in any medium, provided the original work is properly cited. 